\title{
PAGET'S DISEASE OF THE BONE: AN UNUSUAL CAUSE OF LOW BACK PAIN IN AN ADULT MALE
}

\author{
Iulian Resmerita ${ }^{1}$, Cristina Pomirleanu ${ }^{1,2}$, Codrina Ancuta ${ }^{1,2}$ \\ ${ }^{1}$ Rheumatology 2 Department, Clinical Rehabilitation Hospital, Iasi, Romania \\ ${ }^{2}$ Grigore T. Popa University of Medicine and Pharmacy, Iasi, Romania
}

\begin{abstract}
Paget's disease of the bone (PDB) or osteitis deformans is a chronic metabolic bone disorder, characterized by focal increase in bone resorption, followed by a rapid and abnormal bone repair with a disorganized and excessive bone formation, leading to pain, bone deformities and, eventually, fractures. Although the exact etiology of the disease remains controversial, genetic background and environmental factors (viral infections) are typically involved in the complex pathobiology pathways of PDB.

The diagnosis of PDB, subsequent classification in disease subtype (monostotic or polyostotic), activity and severity, and complications (fracture, deformities, osteoarthritis, secondary development of tumors, hearing loss, neural compression, high output congestive cardiac failure) are usually made using a sequence of a blood tests (biochemical markers of bone turnover, especially elevated serum alkaline phosphatase) and imaging (x-ray or bone scan).

Bisphosphonates are widely used for the management aiming to reduce bone turnover, promote healing of osteolytic lesions and improve bone pain.

We report the case of a polyostotic Paget's disease of bone as an unusual case of low back pain.

Keywords: Low Back Pain, Paget Bone Disease, Zoledronic Acid
\end{abstract}

\section{CASE REPORT}

We present the case of a 68 years old male referred to the outpatient rheumatology department in December 2014 with a six-months history of intermittent mechanical low back pain, with a progressive onset, optimally controlled by classic analgesics and/or non-steroidal anti-inflammatory drugs (NSAIDs), but relapsing during drug-free times. His medical background comprises arterial hypertension controlled by calcium blockers combined with sartans, diabetes mellitus treated with biguanides, abnormal lipid metabolism under statin therapy, as well as a basal cell carcinoma surgically removed without complications.

Physical examination revealed a mild lumbar syndrome meaning pain at palpation, paravertebral muscle spasm, limited spine flexibility for flexion (modified Schober test $2 \mathrm{~cm}$ ) and unilateral side; however, neurological exam was normal.

Lab tests were in normal range, except a mild inflammatory syndrome (1.5 times upper normal limit for erythrocyte sedimentation rate, 1.2 times the upper limit of normal for C-reactive protein), mild increase in total serum alkaline phosphatase (2 times upper normal limit for ALP) and lactic dehydrogenase (1.5 times greater than normal range). The routine $\mathrm{X}$-ray of the spine revealed an increased density of the third lumbar vertebral body (L3), with coarsening of the trabecular pattern, accompanied by extensive disc degeneration (L3-L4-L5) (Fig. 1).

We excluded multiple myeloma (normal serum electrophoresis, negative Bence Jones protein, normal skull X-ray), while bone metastasis were overlooked, as well since negative abdominal and thyroid ultrasonography, normal prostate specific antigen, normal chest X-ray.

Technetium 99m (Tc99m) bone scan was further proposed and showed increased radioactive isotope uptake in the L3 but also in the right humerus, without any other affected area (Fig. 2). Additionally, radiological exam of the right humerus confirmed bone hypertrophy of the upper shaft with defor- 


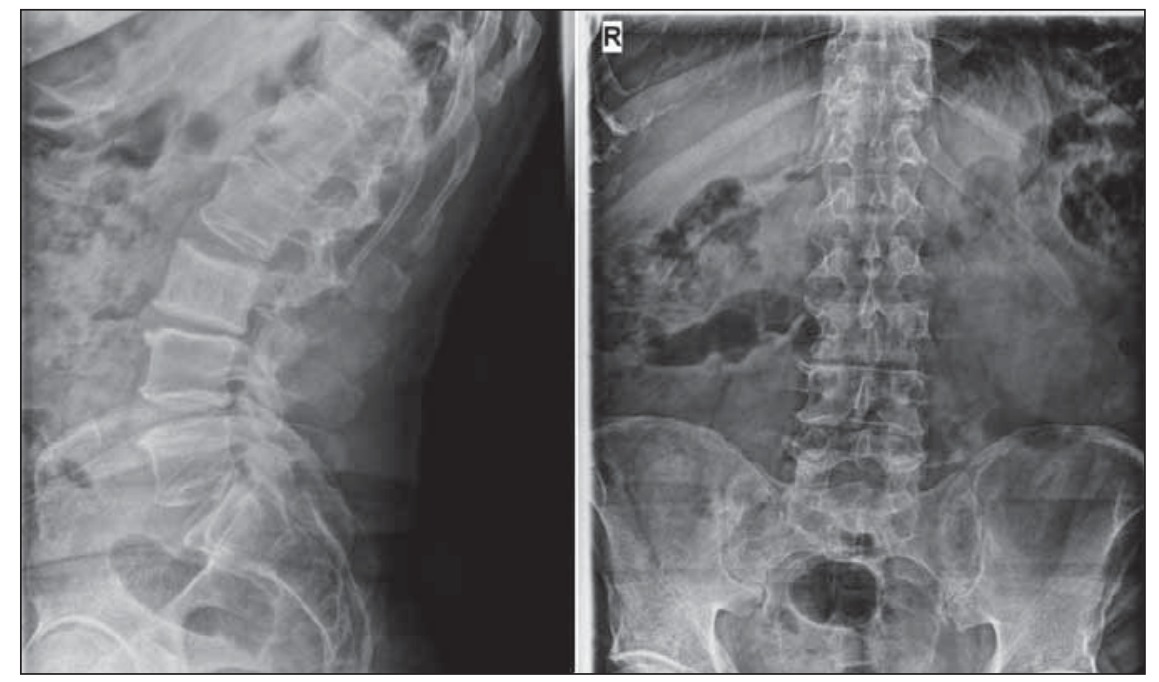

FIGURE 1. Lumbar spine $X$-ray

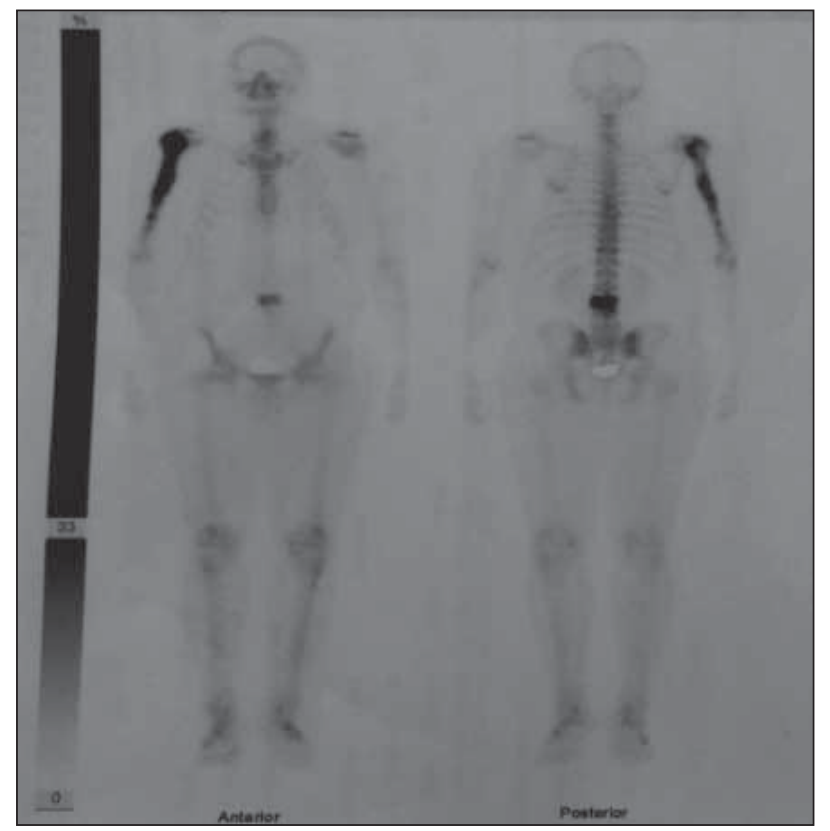

FIGURE 2. Technetium $99 \mathrm{~m}$ bone scan

mity, obviously thickened cortical bone, heterogeneous osteosclerosis and bone demineralization area (Fig. 3).

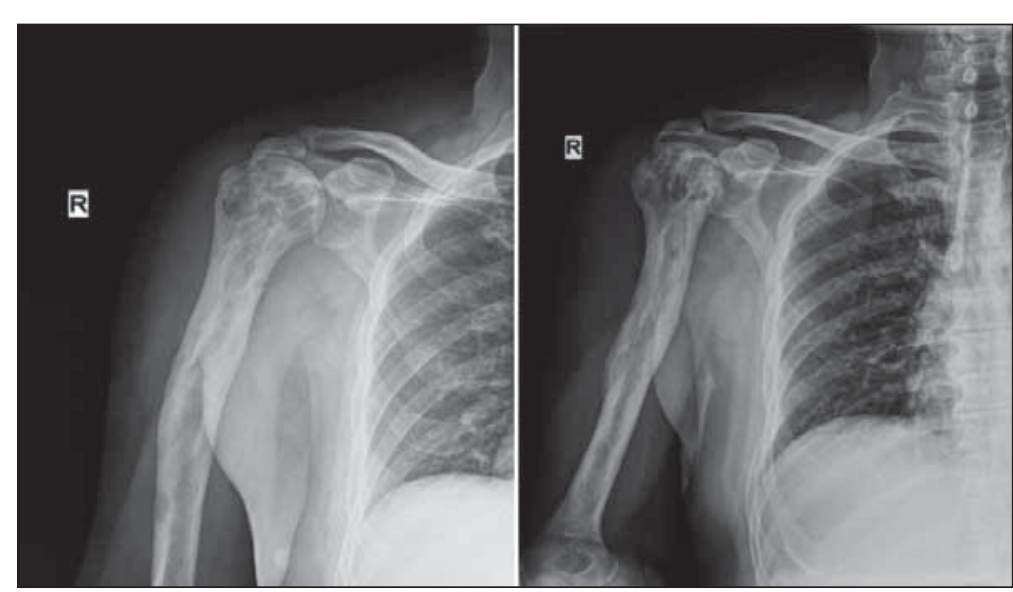

FIGURE 3. Right humerus and shoulder X-rays
Our patient was considered as having a polyostotic subtype Paget's disease of bone and we started zolendronic acid, $5 \mathrm{mg}$ intravenously. 3 months later, meaningful improvement in both clinical and biochemical tests (normal ALP) was reported. Furthermore, Tc99m bone scan performed at 6 months showed significant reduction of pathological bone uptake in the L3 vertebra and right humerus as well, with no new capture region (Fig. 4).

\section{DISCUSSION}

We focused on a patient with low back pain which could be easily classified at first glance as having spine osteoarthritis: chronic intermittent, mild to moderate mechanical pain, with little or no impact on daily activity, good response to simple analgesics and/or NSAIDs, relapsing if drug discontinuation.

A complex hematologic, biochemical and imaging assessment was required in order to establish the correct diagnosis, as the routine spine X-ray showed 


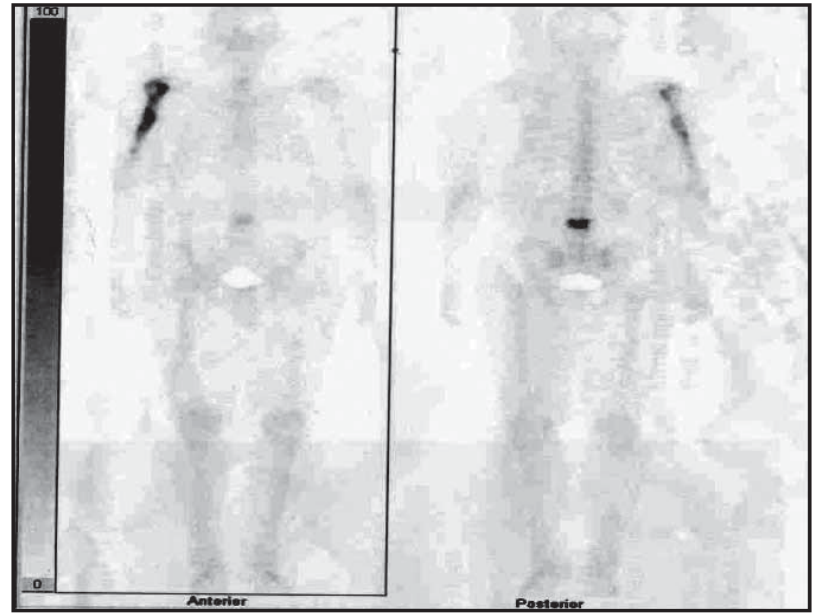

FIGURE 4. Technetium 99m bone scan 6 months after treatment

vertebral lesions (structural changes at the L3 level) compatible with PBD.

Surprisingly, a second pagetic lesion in the humerus, totally asymptomatic, potentially prone to develop complications (fracture or sarcomatous degeneration) was demonstrated by Tc $99 \mathrm{~m}$ bone scintigraphy, an essential technique for staging and monitoring disease activity, as well as treatment efficacy in PBD.

To note, dramatic response with clinical and imagistic remission was reported after 3 , respectively 6 months of zoledronic acid, a new-generation bisphosphonate.

PDB or osteitis deformans is a metabolic bone disease characterized by an excessive rate of bone remodeling, consequent bone expansion with trabecular disorganization, as well as decreased bone strength and quality (1). It is rarely described before 40 , but its prevalence tends to double every decade starting at 50, rising up to $10 \%$ after the ninth decade (2).

\section{Clinical picture}

The clinical spectrum of PDB is highly variable and depends on affected skeletal region, complications (type and magnitude) and metabolic activity (5). The pelvis (70\%), spine (53\%), skull (42\%), and long bones (femur 55\%, tibia $32 \%$, humerus $14 \%$ ) are typically involved, but the disease may concern any skeletal region $(6,7)$.

Pain is the most common symptom described in up to $40-45 \%$ patients, with a specific pattern: usually deep, continuous, precisely located, persistent, with no improvement at rest and increasing during night and with overload $(7,8)$.
Deformities are the second most common manifestation of PDB (12-36\%), occurring mainly in the femur and tibia, causing typical bending (anterolateral in the femur, while anterior in the tibia), gait abnormalities and mechanical stress, with an increased likelihood of joint degeneration $(7,8)$. Skull involvement accounts for circumscribed osteoporosis, followed years later by increases in volume, sclerosis, an enlarged diploe and frontal bossing $(7,8)$.

Complications of PDB might be classified according to specific disease manifestations: skeletal (bone pain, hip and knee osteoarthritis, deformities), cardiovascular (high output heart failure, vascular calcification, valvular stenosis), neurological (deafness, increased intracranial pressure, cranial nerve dysfunction), metabolic (hyperuricemia, hypercalciuria, hypercalcemia, nephrolithiasis) and malignant transformation (osteosarcoma and giant cell tumors) $(8,9)$.

The diagnosis of the disease is commonly made using a sequence of blood tests and imaging (x-rays or bone scans), as follows:

\section{Blood tests}

PDB is associated with excessive bone turnover, confirmed by the elevation of biochemical markers of bone formation and resorption. This increase parallels the magnitude, size, and number of lesions, and might be more pronounced in cases involving the cranium.

Bone-specific isoform of ALP, a sensitive and reliable indicator of bone metabolism, is commonly used for the diagnosis and assessment of disease severity and antiresorptive therapies efficacy. Conversely, osteocalcin, a noncollagenous bone matrix protein and a specific marker of bone formation, has a limited value for the diagnosis and follow-up of PDB as up to $40 \%$ cases present with normal range. Several new biomarkers of bone resorbtion, such as NTX and CTX- $\beta$ have greater diagnostic accuracy (9).

Serum calcium and phosphorus levels are normal in most patients; however, hypercalcemia and hypercalciuria may occur, especially in case of immobilization or fracture $(3,9)$.

\section{Bone imaging}

Scintigraphy reveals the extent of bone involvement in PDB, sites of increased uptake occurring as 
a result of the high rate of bone formation and blood flow. Bone scan with Tc 99-MDP might be positive even before the lytic changes are seen on plain radiography (positive scintigraphy with normal X-ray in $10-15 \%$ cases). Due to the decline in metabolic activity, late stages may show a normal isotope uptake.

Abnormal X-ray comprises hyperostosis, osteosclerosis and bone expansion (10).

Computer tomography (CT) and magnetic resonance imaging (MRI) provide only minimal additional information in the case of uncomplicated PBD, but are suitable in complications such as fractures and sarcomatous degeneration of pagetic bone. Moreover, MRI is considered the gold-standard for the staging of sarcomatous degeneration, as well as for the assessment of neurological complications (spinal nerve roots and cranial nerves compression) $(10,11)$.

\section{Etiology}

The etiology of the PDB is still controversial, although extensive evidence of genetic susceptibility, particularly SQTM1 and genetic polymorphisms of CaSR, ESR1, TNFRSF11B(OPG), TNFRSF11A (RANK), CSF1 (M-CSF),OPTN, TM7SF4 (DCSTAMP), VCP, NUP205, RIN3,PML and GOLGA6A $(1,4,8)$. Environmental factors, viral infections and proinflammatory cytokines might also be involved $(3,4)$.

\section{Treatment}

The management strategies of PDB aim to obtain pain relief and reduce bone remodeling, meaning restoration of bone turnover, rate of bone deposition, reduction of bone vascularization, as well as slowing the progression of the disease $(12,13)$.

Treatment should be recommended for all patients, either symptomatic or asymptomatic, during the preoperative phase of bone surgery, in case of hypercalcemia, if major bones are involved (14), in case of lesions arising in skeletal sites susceptible to complications (cranium, spine), in addition to abnormally high ALP (two to four times above the upper normal limit), eventual comorbidities (13).

Bisphosphonates, particularly nitrogenous ones (alendronate, risedronate, pamidronate, zoledronic acid) are widely used to treat PDB, due to their impact on bone resorption $(13,14)$. Specific regimens for PDB include $40 \mathrm{mg}$ of alendronate daily given for a six months period, $30 \mathrm{mg}$ risedronare daily for two months or intravenous pamidronate at a dose of $30 \mathrm{mg}$ daily for three consecutive days (14). Zoledronic acid is the most potent bisphosphonate FDAapproved for $\mathrm{PDB}$, a single $5 \mathrm{mg}$ intravenous dose resulting in sustained remissions in most patients, which lasts up to two years (14).

Newer agents such as denosumab, a monoclonal antibody against RANKL, are promising for PDB, but still under evaluation (14).

Serum ALP is generally used for the assessment of bisphosphonates efficacy; we talk about biochemical remission when ALP attain and maintain its normal range together with histological evidence of normal bone turnover, while partial remission is defined as a decrease greater than $75 \%$ after three-tosix months of treatment (15). On the other hand, persistent high or an increase in ALP levels usually reflects an active disease. The follow-up should be performed annually, with the indication to restart treatment if ALP begin to rise or in patients displaying a $25 \%$ increase compared to post-treatment levels $(14,15)$.

\section{CONCLUSION}

A complex assessment is commonly recommended in all cases of recurrent mechanical low back pain. As Paget's disease of the bone is the second most common metabolic bone disorder, we suggest a brief evaluation for this disease in all individuals over 50 years old with unexplained elevation of serum ALP, as well as bone pain or deformities. The correct diagnosis, assessment of disease extent and severity and prompt antiresorbtive treatment with newer bisfosphonates are mandatory, with an excellent response even in cases of PDB with multiple site involvement, as was our case.

\section{Consent}

Written informed consent was obtained from the patient for publication of this case report and accompanying images. 


\section{REFERENCES}

1. Britton C., Walsh J. Paget disease of bone - an update. Aust Fam Physician. 2012; 41(3):100-3.

2. Colina M., La Corte R., De Leonardis F., Trotta F. Paget's disease of bone: a review. Rheumatol Int. 2008; 28(11):1069-75.

3. Cundy T., Reid I.R. Paget's disease of bone. Clin Biochem. 2012; 45(1-2):43-8.

4. Chung P.Y., Van Hul W. Paget's disease of bone: evidence for complex pathogenetic interactions. Semin Arthritis Rheum. 2012; 41(5):619-41.

5. Whyte M.P. Clinical practice. Paget's disease of bone. N Engl J Med. 2006; 355:593.

6. Langston A.L., Campbell M.K., Fraser W.D., Maclennan G., Selby P., Ralston S.H. Clinical determinants of quality of life in Paget's disease of bone. Calcif Tissue Int. 2007; 80:1-9.

7. Wermers R.A., Tiegs R.D., Atkinson E.J., Achenbach S.J., Melton L.J. 3rd. Morbidity and mortality associated with Paget's disease of bone: a population-based study. J Bone Miner Res. 2008; 23(6):819-25.

8. Seton M., Moses A.M., Bode R.K., Schwartz C. Paget's disease of bone: the skeletal distribution, complications and quality of life as perceived by patients. Bone. 2011; 48:281.
9. Cundy T., Bolland M. Paget disease of bone. Trends Endocrinol Metab. 2008; 19:246-53.

10. Cortis K., Micallef K., Mizzi A. Imaging Paget's disease of bonefrom head to toe. Clin Radiol. 2011; 66:662-72.

11. Bone H.G. Nonmalignant complications of Paget's disease. J Bone Miner Res. 2006; 21:64-8.

12. Siris E.S., Lyles K.W., Singer F.R., Meunier P.J. Medical management of Paget's disease of bone: indications for treatment and review of current therapies. J Bone Miner Res. 2006; 21(2).

13. Reid I.R. Pharmacotherapy of Paget's disease of bone. Expert Opin Pharmacother. 2012; 13(5):637-46.

14. Luiz Griz, Daniele Fontan, Patricia Mesquita et al. Diagnosis and management of Paget's disease of bone. Arq Bras Endocrinol Metab. 2014; 58(6):587-99.

15. Selby P.L., Davie M.W., Ralston S.H., Stone M.D. Guidelines on the management of Paget's disease of bone. Bone. 2002; 31:366-73. 